\title{
Transformations, Recrystallization, Microtexture and Plasticity in Titanium Alloys
}

\author{
Shanoob Balachandran ${ }^{1,2}$, Abhishek Tripathi ${ }^{1,2}$, Arunima Banerjee ${ }^{1,3}$, Manaswini Chinara ${ }^{1,4}$, Ravi Teja, 1,5 \\ S.J. Suresha ${ }^{1,6}$, Deep Choudhuri ${ }^{7}$, Rajarshi Banerjee ${ }^{7}$ and Dipankar Banerjee ${ }^{* 1}$ \\ ${ }^{1}$ Materials Engineering, Indian Institute of Science, Bangalore, India \\ ${ }^{2}$ Currently at Max-Planck Institute fur Eisenforschung, Germany \\ ${ }^{3}$ Currently at John Hopkins University, USA \\ ${ }^{4}$ Currently at Tata Steel, R\&D, Jamshedpur, India \\ ${ }^{5}$ Currently at National Minerals Development Corporation, India \\ ${ }^{6}$ Currently at CENSE, Indian Institute of Science, Bangalore, India \\ ${ }^{7}$ Department of Materials Science and Engineering, University of North Texas, USA \\ * Corresponding author: dbanerjee@iisc.ac.in
}

\begin{abstract}
The objective of this contribution is to summarise the range of $\alpha$ crystallography and distribution that arises from the $\beta$ to $\alpha$ transformation and the hot working of $\alpha / \beta$ alloys and their recrystallisation and relate these features to the slip character of these alloys. $\alpha$ morphology and distribution have been mapped as a function of $\beta$ stabilizer content and aging temperature. A dominant transgranular grouping of $\alpha$ variants with a common close packed direction characterizes transformation patterns across the entire range of $\beta$ stabilizer content. Recrystallization during thermomechanical processing in the $\alpha / \beta$ regime has been studied with the help of fine scale, orientation image mapping techniques. Two distinct recrystallization processes have been identified. The first is associated with the well-known $\alpha$ globularisation process. An alternative recrystallization process has also been described in which newly recrystallized $\alpha$ and $\beta$ grains form with the Burgers orientation relationship with each other. This process has been called epitaxial recrystallization. The role of transformation and recrystallisation texture on the plasticity of titanium alloys is assessed through quantitative EBSD /TEM based analysis of slip systems in the $\alpha$ phase and slip transfer between the $\alpha$ and $\beta$ phases.
\end{abstract}

\section{Introduction}

In this contribution we consolidate examples from recent, diverse studies on the transformation [1-3] and deformation behaviour [4-6] of $\alpha / \beta$ titanium alloys to illustrate relationships between local texture and the fine scale structure of slip in $\alpha / \beta$ titanium alloys. The paper first summarises briefly the understanding that has emerged in recent years of $\alpha$ morphology, distribution, and crystallography arising from the $\beta$ to $\alpha$ transformation and hot working in the $\alpha+\beta$ regime. While sideplate growth from grain boundary $\alpha$ results in colonies of similarly oriented variants in $\beta$ lean alloys, transgranular colony structures arise from a branching of $\alpha$ laths when the transformation occurs at high temperatures relative to the $\beta$ transus. A stress accommodation assisted grouping of $\alpha$ laths with a common close packed direction dominates transgranular structure across the entire range of $\alpha / \beta$ compositions, irrespective of specific alloy content and heat treatment. As is well known, hot working of $\alpha / \beta$ alloys with colony structures can result in groups of $\alpha$ grains that are similarly oriented, arising out of the conventional globularisation process. It has more recently been shown that under certain conditions an 'epitaxial' recrystallisation process results in equiaxed $\alpha$ and $\beta$ that are Burgers related. Since the Burgers orientation relationship allows a parallelism of certain slip planes and directions in the $\alpha$ and $\beta$ phases, these variations in $\alpha$ distribution and crystallography result in characteristic slip features that are described based on EBSD/ TEM analysis of slip systems.

\section{Experimental}

The alloys that are reported in this study are Ti-Mo alloys, Ti-6Al-2Sn-4Zr-2Mo (Ti6242) and Ti-5Al-5Mo-5V$5 \mathrm{Cr}$ (Ti5553). A range of compositions was assessed for transformations in the Ti-Mo system using a LENS processed compositionally gradient structure. Ti6242 and Ti5553 were provided by Pratt and Whitney and DMRL respectively. Electron backscatter diffraction (EBSD) has been used extensively to characterise local texture, as 
well as determine slip systems from slip offsets on samples deformed in compression. In addition, TEM based orientation imaging using precession electron diffraction (OIM-PED) using the Nanomegas system has been used in some cases to examine fine scale orientation effects at resolutions below the level that could be achieved by EBSD. Details of these methods as applied to these studies have been reported elsewhere [2-6] and will not be repeated here. Heat treatments and processing conditions can be found in [1-6].

\section{The $\beta$ to $\alpha$ transformation and slip lengths}

The effect of $\beta$ stabiliser content on the morphology and distribution of $\alpha$ phase has been explored through the effect of Mo content on transformation structures [1]. Examples of microstructures formed in alloys with different $\beta$ stabiliser contents and cooling rates are shown in Figure 1. Figure 1a shows structures typical of low $\beta$ stabiliser content alloys with slow cooling rates from the $\beta$ phase region where the entire structure is dominated by widmanstatten $\alpha$ side plates emanating from grain boundary $\alpha$ to form colony structures. With higher $\beta$ stabiliser content or higher cooling rates (Figure1 b-d) transgranular $\alpha$ increasingly dominates the structure. The relatively small, interweaving transgranular colonies shown in Figure $1 \mathrm{~b}$ and $\mathrm{c}$ arise from a branching process of transgranular $\alpha$ laths that has been described in [1]. As shown in [1,2], transgranular $\alpha$ consists of groups of three $\alpha$ variants that share a common [11 20$]$ direction, and this configuration arises from 4 sets of $12 \alpha$ variants that arise out of the Burgers orientation relationship $\{110\} / /(0001) ;<111>/ /<11 \overline{2} 0>$ each of which share a common parent $<111>\beta$ direction. This feature of transgranular $\alpha$ structure is observed irrespective of the alloying content as illustrated in the examples of Figure 2 and 3 which show such variant groups in structures that are representative of Figure1b and Figure1d respectively.

In alloys with high volume fraction $\alpha$ and slow cooling rates from the $\beta$ phase field the slip length is defined by the colony size as illustrated in Figure 4. Shear is initiated in $\alpha$ and several of these slip planes and directions in identically oriented, parallel $\alpha$ variants are coplanar or nearly so with those in the thin ribs of retained $\beta$ in such structures $[7,8]$. The slip lengths for the $\alpha$ configurations shown in Figure 1b, c, and $d$ are less obvious since slip transfer across multivariant $\alpha$ distributions such as these has not been investigated thus far. For these structures it is tempting to assume that the slip length is restricted to the individual variant thickness or at the most the few similarly oriented variant groups that might exist in such a distribution. However this is not the case. Figure 5a indicates that slip lines can extend across a group of multivariant distributions that exist with the common [11 $\overline{2} 0]$ direction. EBSD analysis shows that these slip lines are consistent with coplanar $(0001)$ and $\{10 \overline{1} 1\}$ slip planes in the three variants constituting the group with the common $[11 \overline{2} 0] \alpha / /<111>\beta$ direction as the Burgers vector (Figure $5 b$ ). The effective slip length is therefore equivalent to the 'colony' size where the 'colony' now represents the size of these specific multivariant groups with a common [11 $\overline{2} 0]$ direction rather than the size of a group of similarly oriented variants shown in Figure 4.

Figures $6 \mathrm{a}$ and $\mathrm{b}$ provide an example of restricted slip lengths associated with the multivariant distribution of $\alpha$ in a relatively high volume fraction $\beta$ matrix shown in Figures $1 \mathrm{~d}$ and 3. The origin of such slip length restrictions have been investigated by transmission electron microscopy (Figure 6). Slip initiates in the $\beta$ phase and has been identified though Burgers vector and slip plane analysis to belong to the $\{112\}<111>$ system. Figure 7a shows that for a particular set of this family, slip planes and directions in the twelve variants of the $\alpha$ phase will lie close to the $\beta$ slip system in only a few cases and these are associated with high Luster-Morris parameters, $\mathrm{m}^{\prime}$ [9], defined by the product of the cosine of the angles between slip plane normal and slip directions between the $\alpha$ and $\beta$ phases for each $\alpha$ variant (Figure 7b). Figure 7c and d shows an example of slip transfer across an $\alpha$ plate and blocked slip bands. The slip length will then be defined by the non-random distribution of the $\alpha$ variants (Figure 3 and reference [2]) and the probability of slip bands in $\beta$ intersecting variants across which slip transfer cannot occur.

\section{Hot worked and recrystallised $\alpha+\beta$ structures}

\section{Equiaxed $\alpha$ and transformed $\beta$}

The evolution of microtexture with specific reference to the variation of the orientation relationship between $\alpha$ and $\beta$ with hot working and recrystallisation has recently been examined [3]. The classic globularisation process of $\alpha$ laths with hot working involves the formation of subgrains in the $\alpha$ and $\beta$ phases, increasing misorientation 


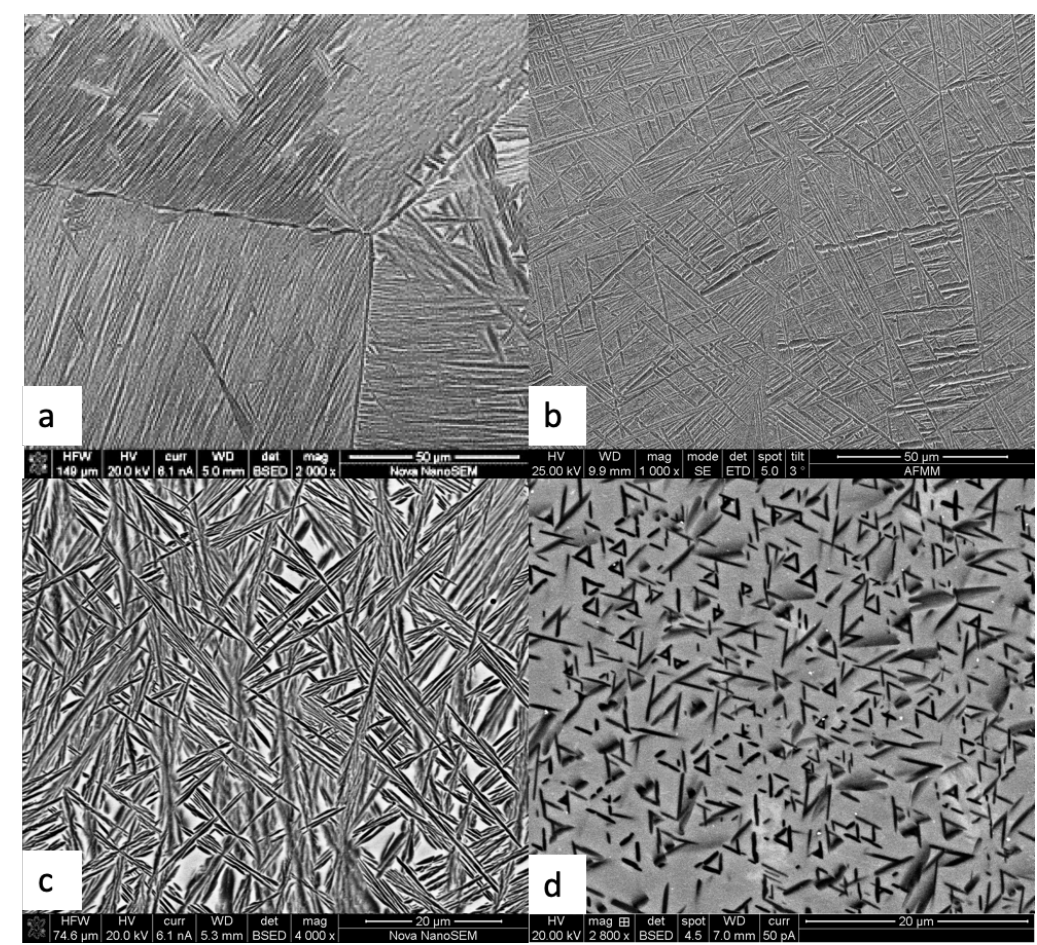

Figure 1: The variety of $\alpha$ morphologies in Ti-Mo alloys with increasing Mo content from (a) to d)
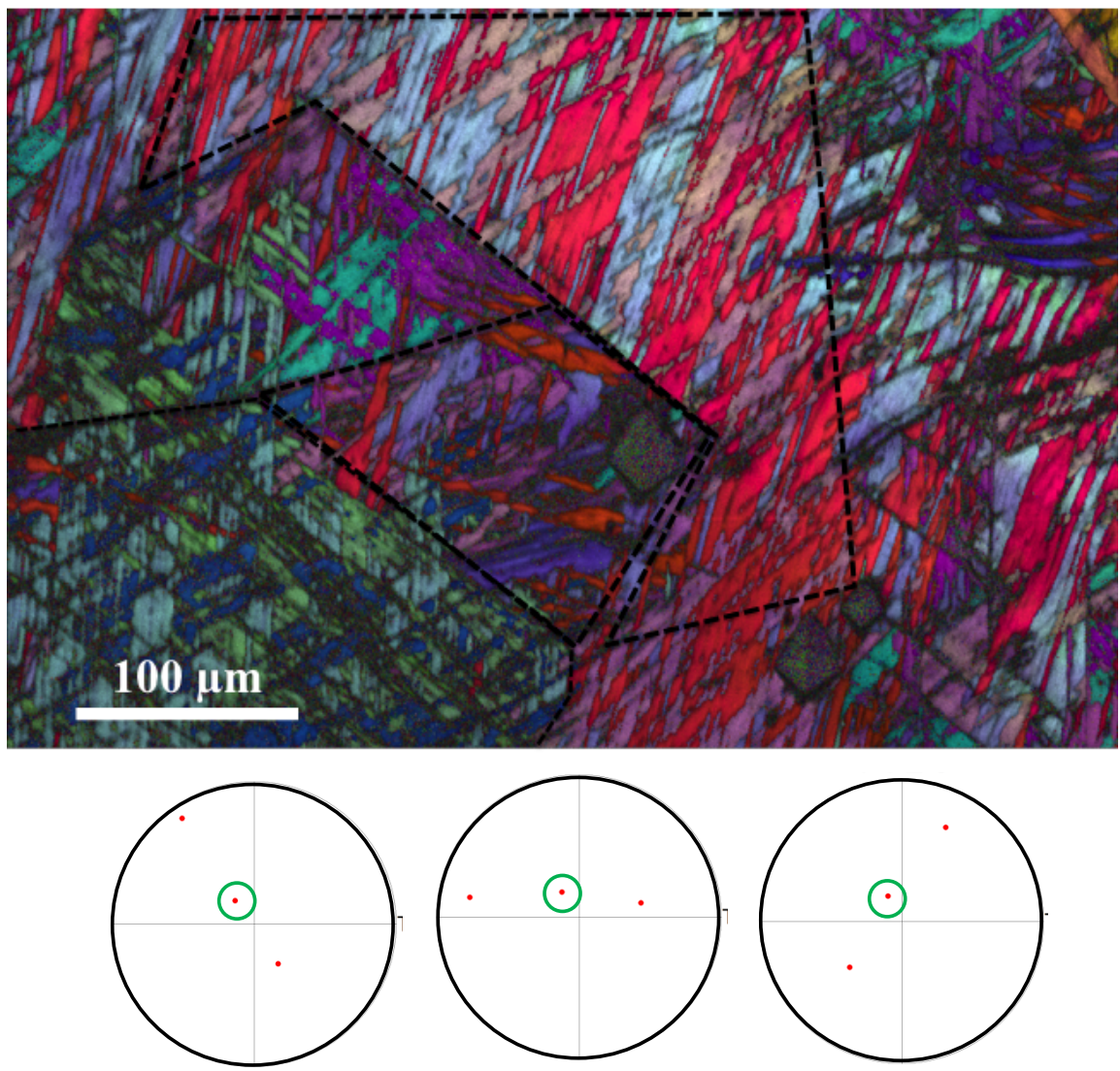

$11 \overline{2} 0$ poles

Figure 2: EBSD of areas such as in Fig. 1b in a Ti6242 alloy air cooled from above the $\beta$ transus. The dark lines outline approximately areas of similar groups of $\alpha$ variants that share a common $11 \overline{2} 0$ pole. Pole figures of one such area show the 3 variants with the common pole encircled by the green circle. 


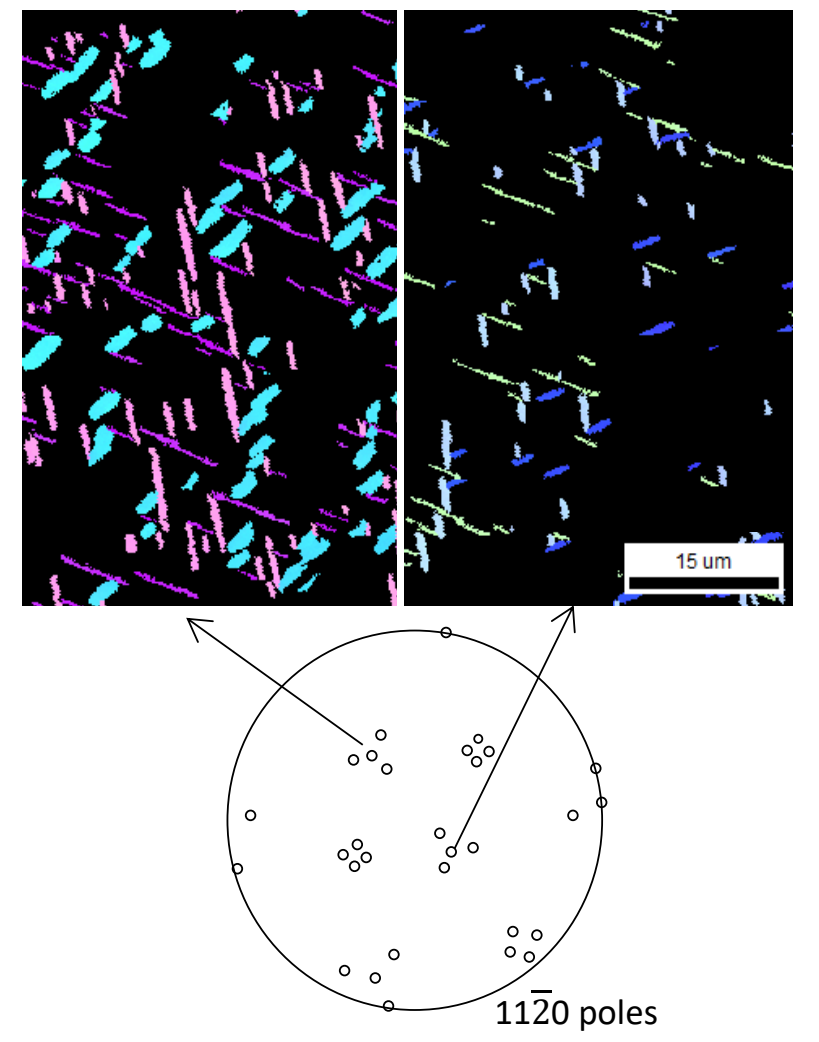

Figure 3: EBSD of areas such as in Fig. 1d in a Ti5553 alloy and associated pole figure from all $12 \alpha$ variants present in this area. The two orientation images have been partitioned to show that variants arranged in a triangular fashion share a common pole. Figure reproduced with permission from [2].

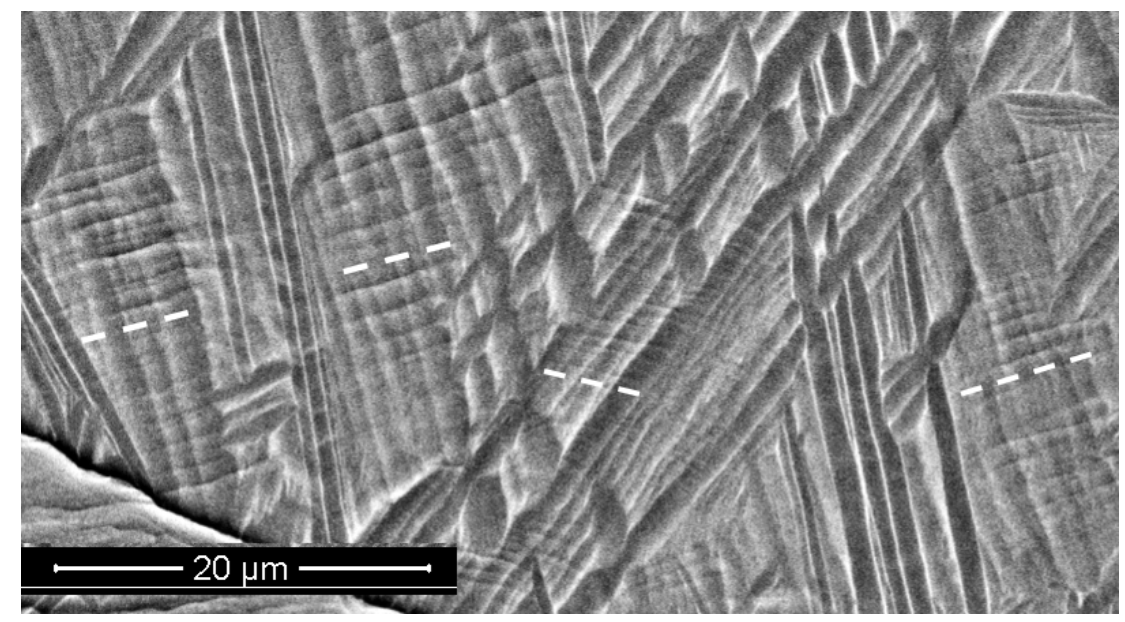

Figure 4: Surface slip offsets after compression at room temperature shows slip length restricted by colony size in a $\beta$ heat treated Ti6242 alloy. The traces of slip planes in each colony are indicated by dashed lines. 


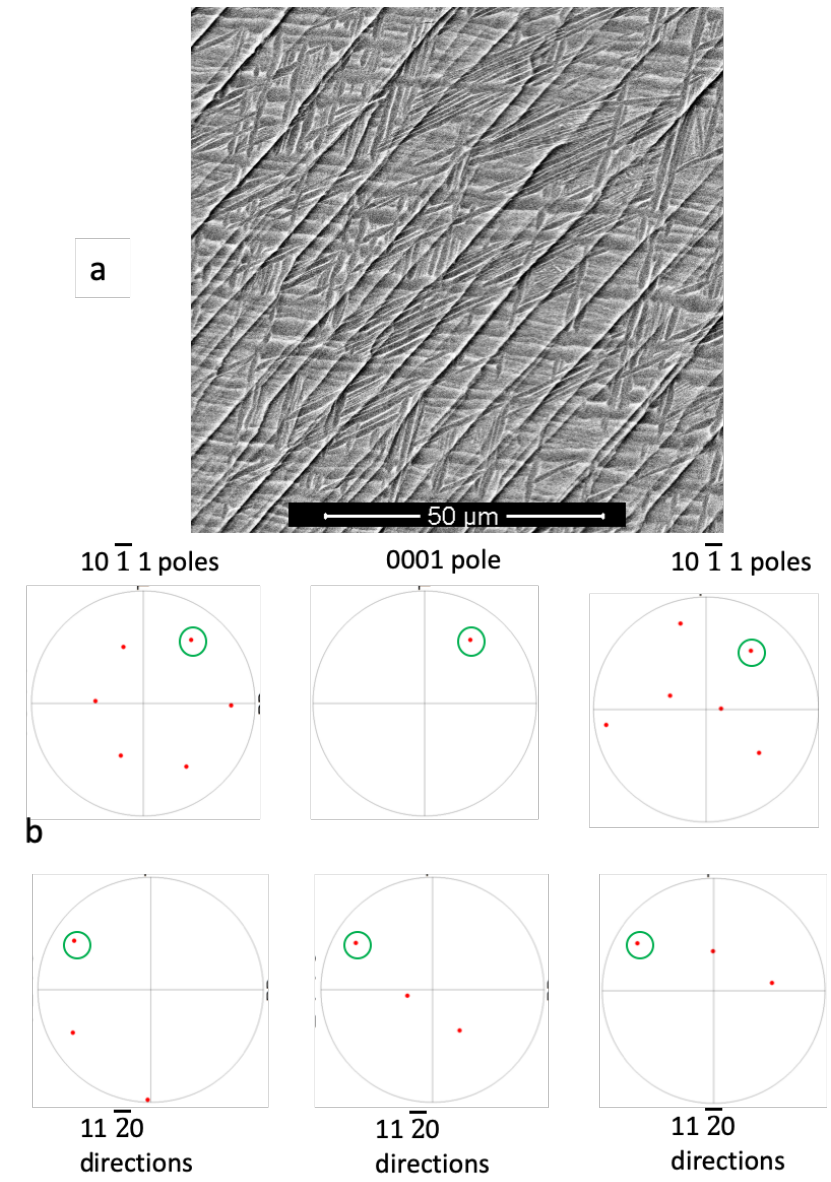

Figure 5: (a) Slip transfer across multivariant $\alpha$ structures in a Ti6242 (b) EBSD of areas such as in (a) shows the slip bands to be associated with parallel or nearly parallel $\{10 \overline{1} 1\}$ and (0001) planes with the common slip vector $[11 \overline{2} 0]$ of the 3 variants that constitute this multivariant region (see also Fig. 2).

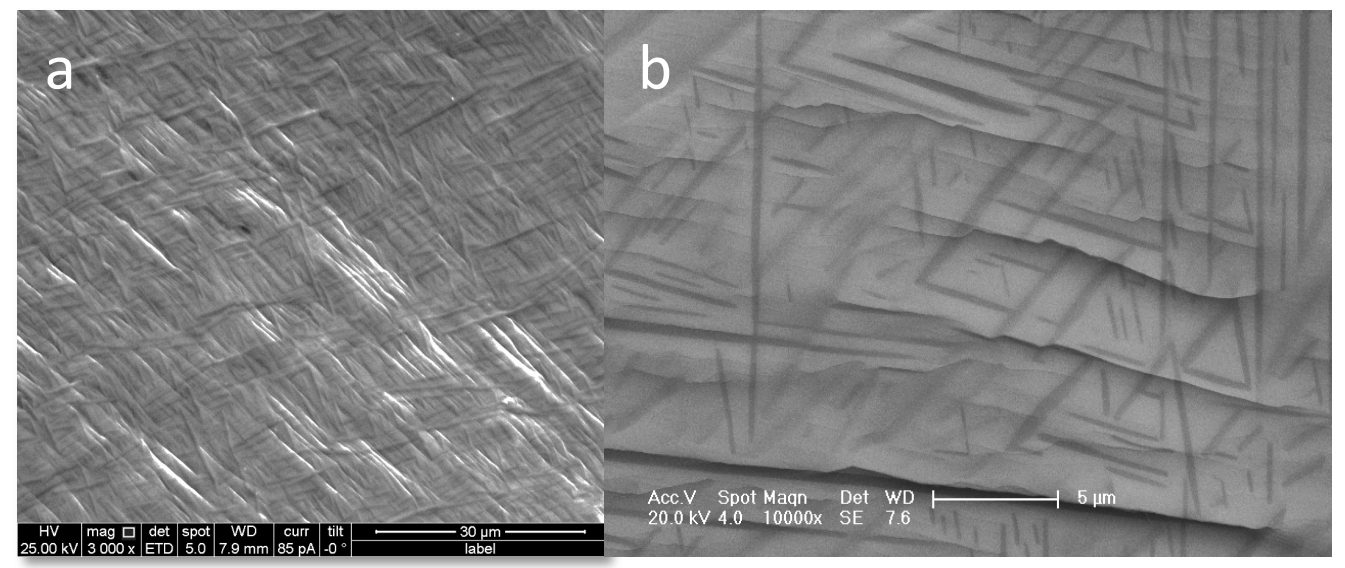

Figure 6: Surface slip offsets after compression at room temperature in Ti5553 (a) restricted slip lengths in the multivariant structure (b) detail of (a) showing that slip lines shear some $\alpha$ plates but are blocked at others. Slip parallel to $\alpha / \beta$ interfaces is also observed. 


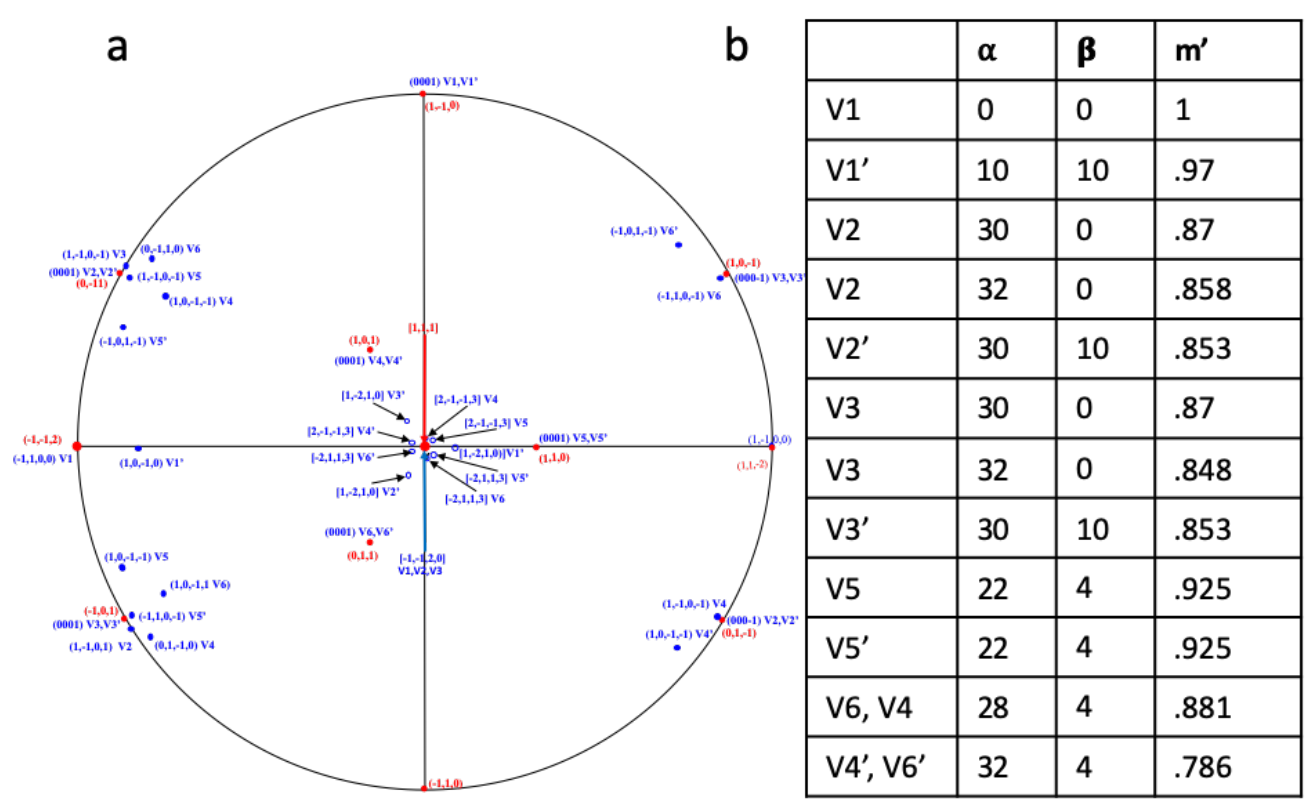

C

d

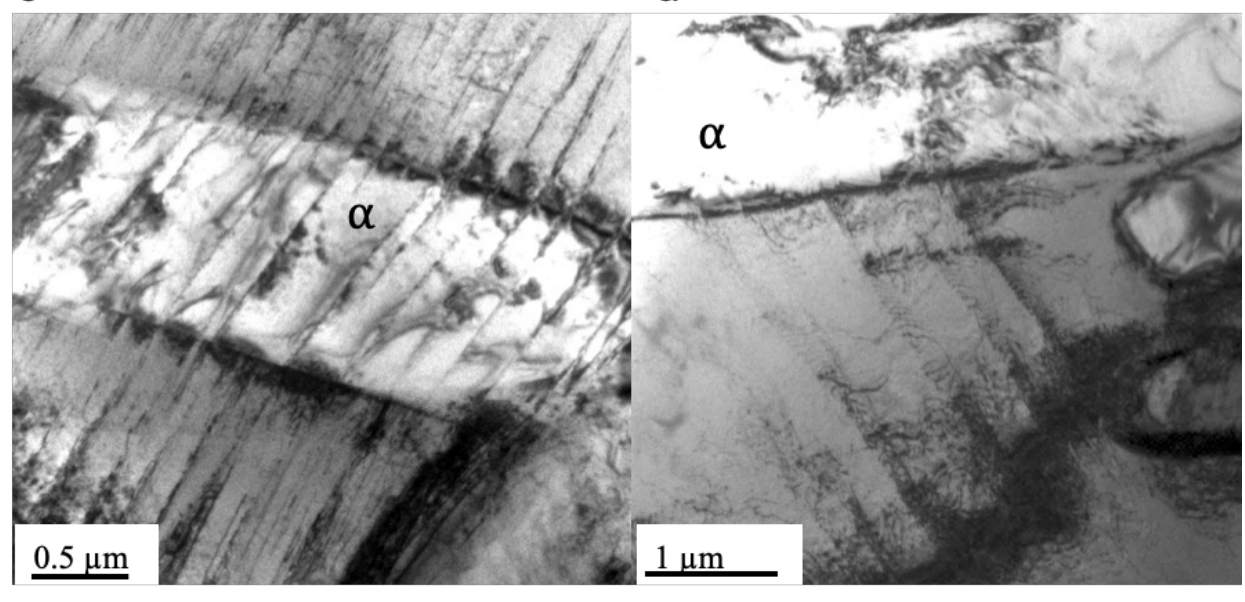

Figure 7: Slip transfer from $\beta$ to $\alpha$ in multivariant $\alpha$ structures in a Ti5553 alloy. The slip system in $\beta$ has been identified as $\{112\}<111>$. Various possible slip systems in 12 variants of the $\alpha$ phase are shown in the stereographic projection in (a) identified as $\mathrm{Vi}^{\text {and }} \mathrm{Vi}^{\prime}$ etc where the system marked with a prime shares a common basal plane with the variant without a prime (b) The associated Luster-Morris parameters (c) slip transfer for the $\mathrm{V1}^{\prime}$ variant (d) blocked slip bands 
between the subgrains with strain and finally the pinching of $\alpha$ to form equiaxed morphologies $[10,11]$. This process is akin to geometric dynamic recrystallisation [12] (in the sense that there is no migration of subgrain boundaries) and is illustrated in Figure 8. As is clear from the figure, each $\alpha$ subgrain is increasingly misoriented from the surrounding $\beta$ grains as the misorientation increases. Nevertheless, even at the point of complete pinch off, as between $\alpha$ grains 1,2 and 3, the original Burgers orientation relationship can still be discerned, and the two similarly oriented $\alpha$ laths retain similarities in their orientations. It is this process that underlies the formation of macrozones of $\alpha$ orientations that arise from processing a structure consisting of colonies of similarly oriented variants, as described for example in [13] and shown as an example in Figure 9. The implications of the existence of such macrozones for dwell fatigue is well known [14].

If, on the other hand, classical recrystallisation of the $\alpha$ and $\beta$ phases occurs, then the $\alpha$ and $\beta$ grains have been found to recrystallise in a Burgers orientation relationship with each other. This has been called 'epitaxial recrystallisation', illustrated in Figure 10. Figure 10b shows recrystallised $\beta$ and $\alpha$ grains all which are Burgers oriented with respect to each other. This structure is realised from a starting multivariant $\alpha$ distribution in a single large sized $\beta$ grain, as in Figure 1d. Figure 11 shows that the sharp transformation texture arising out of the Burgers relationship tends towards randomisation by globularisation, but the Burgers relationship is again restored on an average by the epitaxial recrystallisation process. The observation of such epitaxial recrystallisation processes was possible in the Ti5553 alloy since the orientation of recrystallised $\beta$ grains is easily detected as the phase is retained on rapid cooling from the hot deformation or recrystallisation anneal temperature in this metastable $\beta$ alloy. As a consequence of this process, equiaxed $\alpha$ may well share a Burgers orientation relationship with some the $\beta$ grains that surround it, a feature of microtexture that has not been generally recognised in the past.

This has consequences for the plasticity of equiaxed structures. Figure 12a shows slip offsets in a bimodal Ti6242 alloy strained in compression. Slip lines are seen in equiaxed $\alpha$ and in transformed $\beta$. A significant fraction of slip in transformed $\beta$ arises from slip transfer from equiaxed $\alpha$ (which yields first) into the transformed $\beta$, an example of which is provided in the inset to Figure 12a. EBSD based analysis (not shown here) has been used to obtain Schmid factors of slip systems observed in the colony structures of transformed $\beta$. As indicated in Figure $12 \mathrm{~b}$ and $\mathrm{c}$, the slip systems observed in majority of the cases is not that due to a globally high Schmid factor arising from the compression axis, but occurs with a high Luster-Morris parameter associated to the slip system in equiaxed $\alpha$ and the adjacent colony structure, implying that that slip transfer dominates the spread in plasticity into the transformed beta structure. This is possible because in each case of slip transfer, the equiaxed $\alpha$ is near Burgers oriented to the underlying $\beta$ grain of the transformed $\beta$, thereby ensuring parallelism of slip planes in the colony $\alpha$ and equiaxed $\alpha$ through the Burgers orientation relationship.

\section{$\beta$ grain misorientations and grain boundary $\alpha$}

The epitaxial recrystallisation process also results in special misorientations between the recrystallised $\beta$ grains, explained in [3]. As pointed out by Cayron [15], the reverse transformation from $\alpha$ to $\beta$ will result in $\beta$ variants that will be misoriented with respect to each other by a set of special axis/angle relationships given in Table 1 .

Table 1. Special misorientations between $\beta$ grains [15]

\begin{tabular}{|l|l|}
1 & $\begin{array}{l}{[111] / 60^{\circ}} \\
\text { equivalent to } \\
{[011] / 70.5^{\circ}}\end{array}$ \\
\hline 2 & {$[011] / 60^{\circ}$} \\
\hline 3 & {$[011] / 49.5^{\circ}$} \\
\hline 4 & {$[011] / 10.5^{\circ}$} \\
\hline
\end{tabular}

Since the epitaxial recrystallisation process described above results in recrystallised $\beta$ grains that are Burgers oriented to $\alpha$ when hot deformation is carried out in the two phase region, many of these $\beta$ grains are then oriented with respect to each other by these special relationships when they grow and meet. Figure 13 shows examples of such grains in $\alpha+\beta$ recrystallised structures. The $\beta$ grain misorientations are particularly easy to analyse in metastable $\beta$ alloys such as Ti5553 since the $\beta$ phase is retained on quenching from heat treatment temperatures. The existence of special misorientations between $\beta$ grains has two important consequences. The first relates to the formation of grain boundary $\alpha$. It has been shown that grain boundary $\alpha$ forms preferentially at those boundaries that are related by these misorienations [16]. This is because their crystallography allows the $\alpha$ phase formed at 
such $\beta$ boundaries to be Burgers oriented to both grains [17], as schematically shown in Figure 13a. Since Widmansttaten
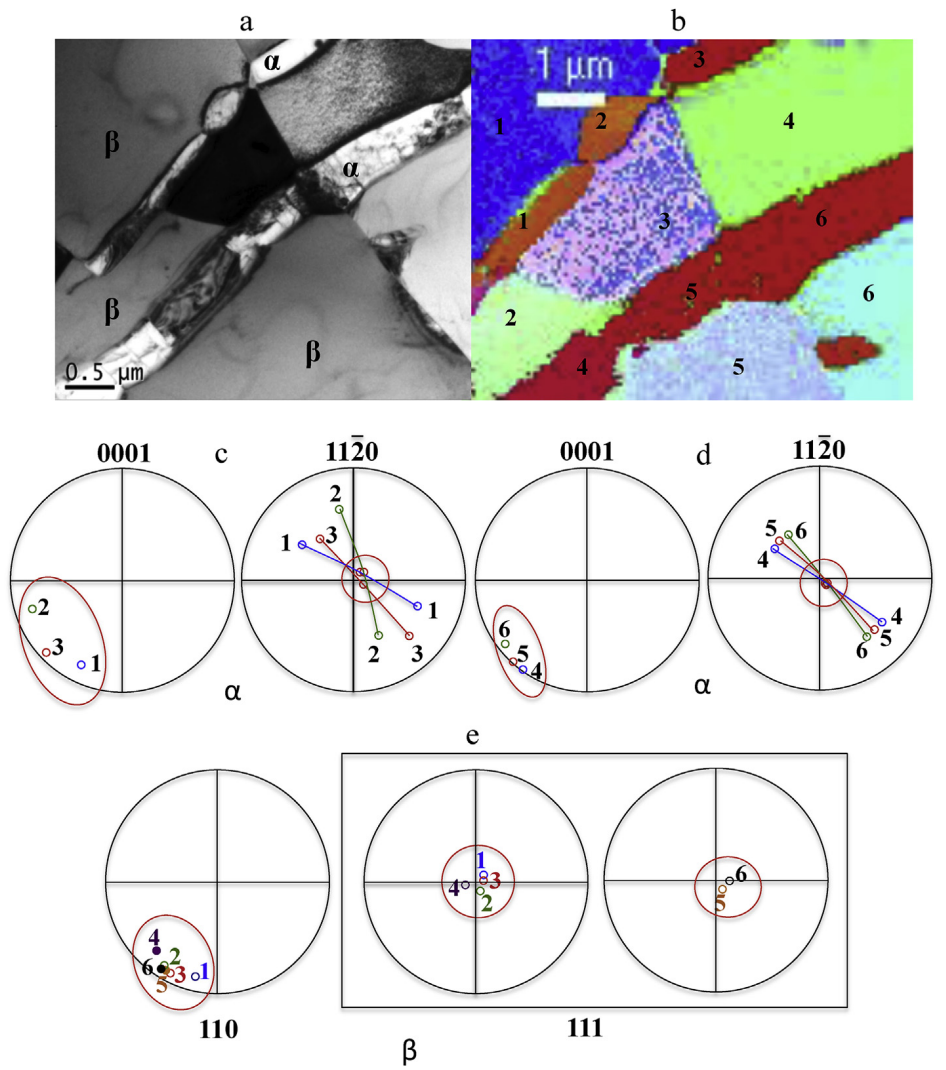

Figure 8: Globularisation in Ti 5553 (a) bright field image showing cusps formed at $\alpha$ and $\beta$ subgrain boundaries (b) OIM-PED of this area (c) pole figures of the grains marked in (b) showing misorientations of the $\alpha$ and $\beta$ subgrains. The original Burgers relationship is shown by the red circles. The misorientations in this case are primarily about that $<11 \overline{\mathbf{2 0}}>$ pole parallel originally to $<111>\beta$. Figure reproduced with permission from [3].

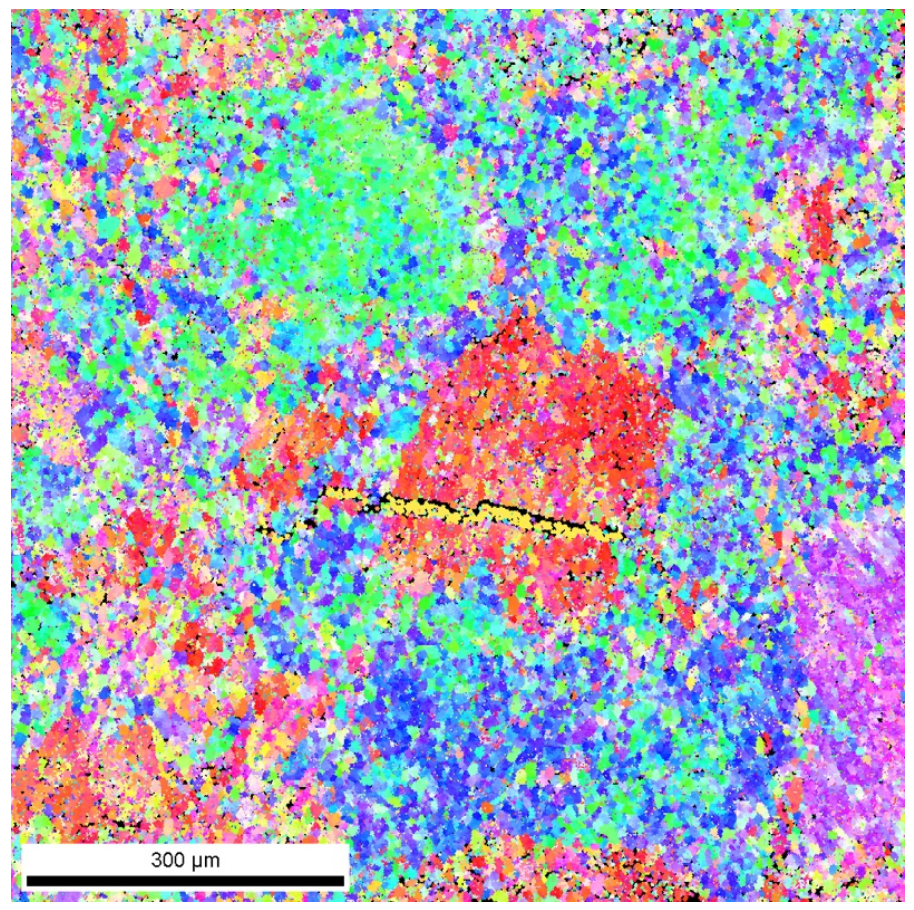

Figure 9: Macrozones formed by globularisation of colony structures in a Ti6242 alloy and a dwell fatigue crack associated with such macrozones. Each such macrozone consisting of similarly oriented $\alpha$ originates from a colony of similarly oriented $\alpha$ variants in the structure prior to hot deformation. Figure courtesy Amit Bhattacharjee and J.C. Williams, Ohio State University, USA. 

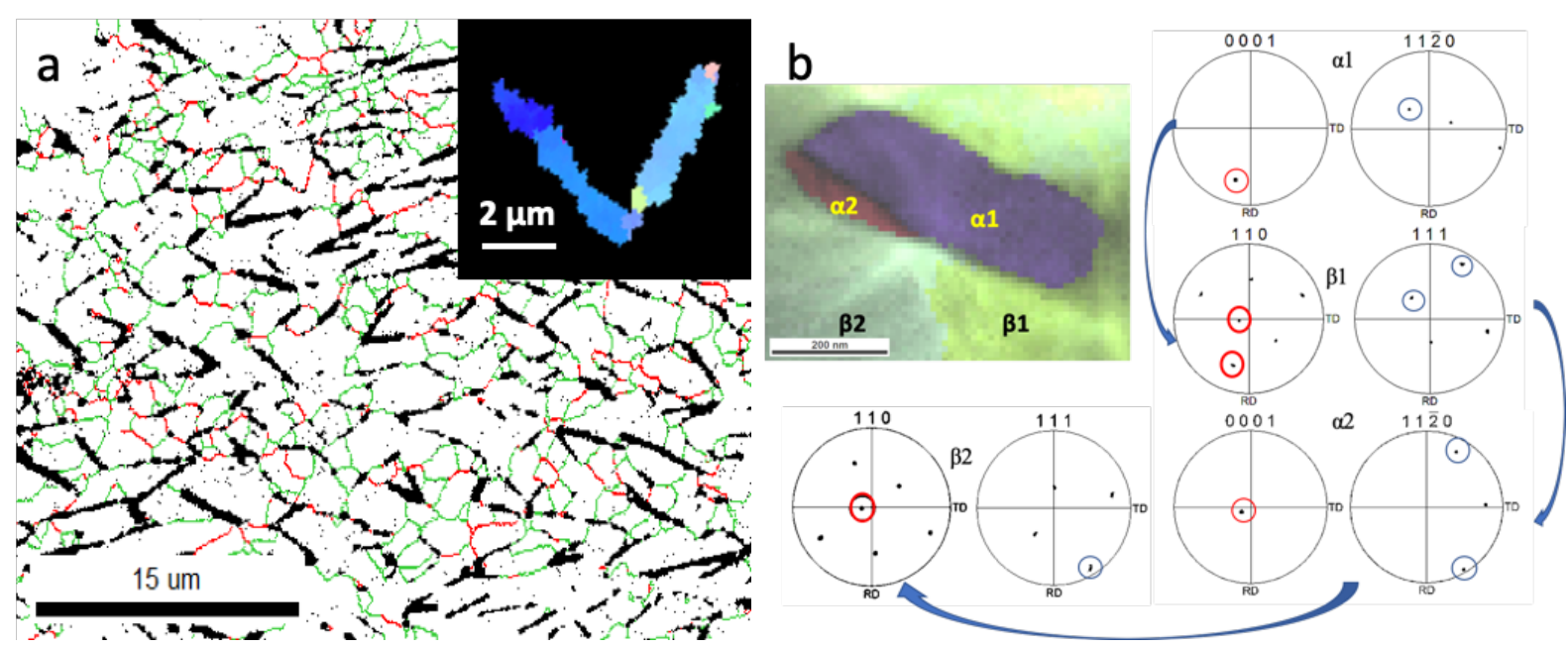

Figure 10: Epitaxial recrystallisation in Ti5553. The original structure had $\alpha$ plates within a single $\beta$ grain in the starting structure (as in Fig 1d) prior to hot deformation (a) shows EBSD with phase partitioning. The $\alpha$ plates are dark and high angle grain boundaries in $\beta$ are in red. The inset shows high angle boundaries within $\alpha$ plates. The OIM-PED image shows two $\alpha$ grains formed by recrystallisation of a single $\alpha$ lath and $2 \beta$ grains. The pole figures show that the $\alpha 1$ grain is Burgers related to the $\beta 1$ grain while the $\alpha 2$ grain is Burgers related to both the $\beta 1$ and $\beta 2$ grains. Figure reproduced with permission from [3].

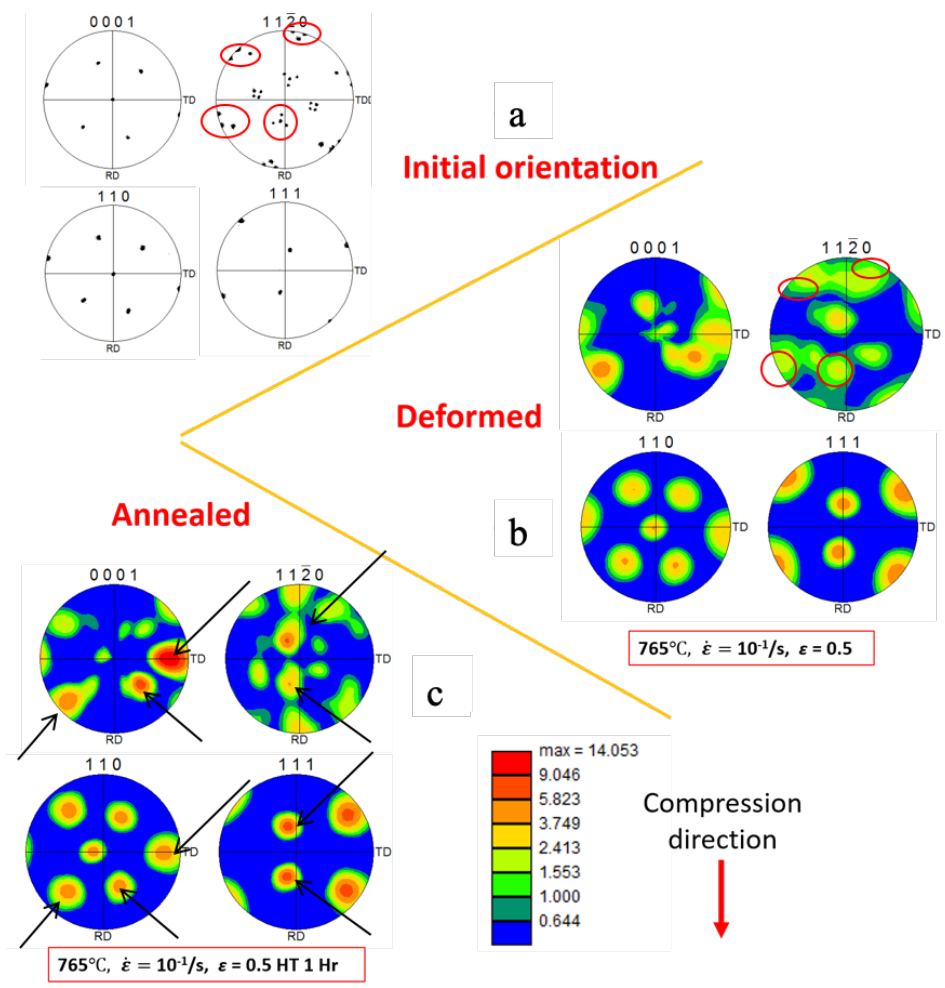

Figure 11: Epitaxial recrystallisation in Ti5553. The same area has been tracked in the hot deformation and annealing process. The sharp transformation texture in (a) has been randomised by globularisation in (b) by hot deformation (c) The Burgers relationship is restored on an average compared to (b) on annealing by epitaxial recrystallisation as marked by arrows showing the parallel poles in the $\alpha$ and $\beta$ phases. Figure reproduced with permission from [3]. 

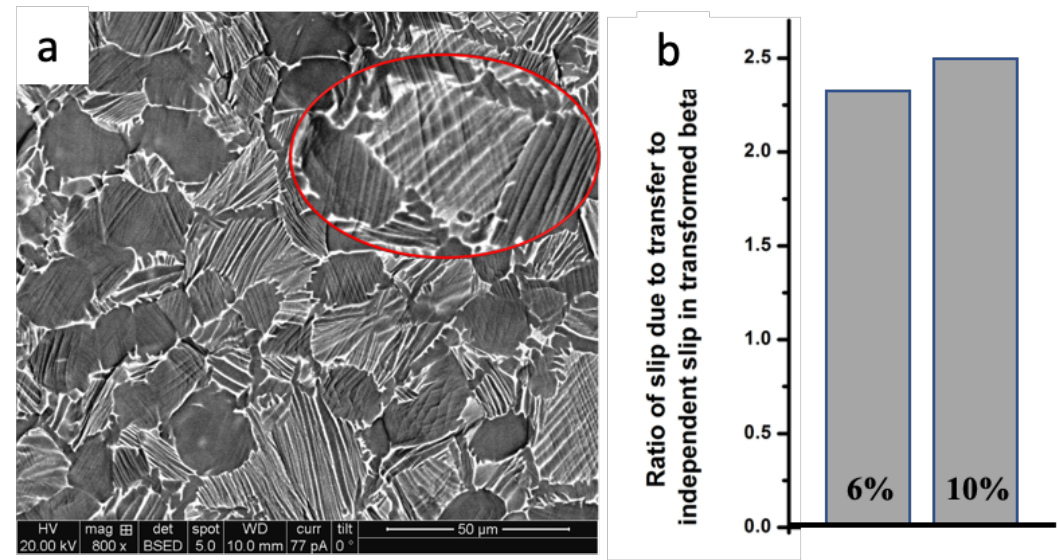

\begin{tabular}{|c|c|c|c|}
\hline C & Transformed $\boldsymbol{\beta}$ & $\begin{array}{c}\text { Slip system } \\
\text { independently activated }\end{array}$ & $\begin{array}{c}\text { Slip system activated } \\
\text { due to slip transfer }\end{array}$ \\
\hline $10 \%$ (42 grains) & 12 & 30 \\
\hline $6 \%$ (40 grains) & 12 & 28 \\
\hline
\end{tabular}

Figure 12: Slip transfer between equiaxed $\alpha$ and $\alpha$ colonies in transformed $\beta$ in a bimodal structure of Ti 6242 alloy (a) shows surface slip offsets in the alloy deformed in compression at room temperature. The inset is at a higher magnification showing parallel slip planes in equiaxed $\alpha$ and a $\alpha$ colony (b) the ratio of the number of slip bands that are activated in colony $\alpha$ due to slip transfer to independent activation of slip at two different strain levels (c) the table shows the numbers of grains and actual number of slip systems measured.

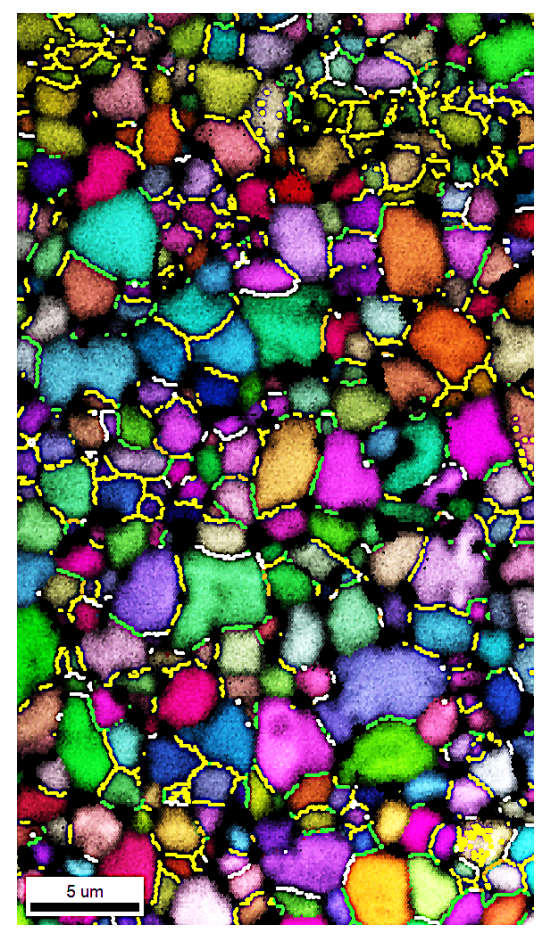

Figure 13: Recrystallised structure in Ti5553. The phase partitioned image shows equiaxed $\alpha$ in black. The boundaries between $\beta$ grains with special misorientations are indicated by thick lines. Figure reproduced with permission from [3]. 

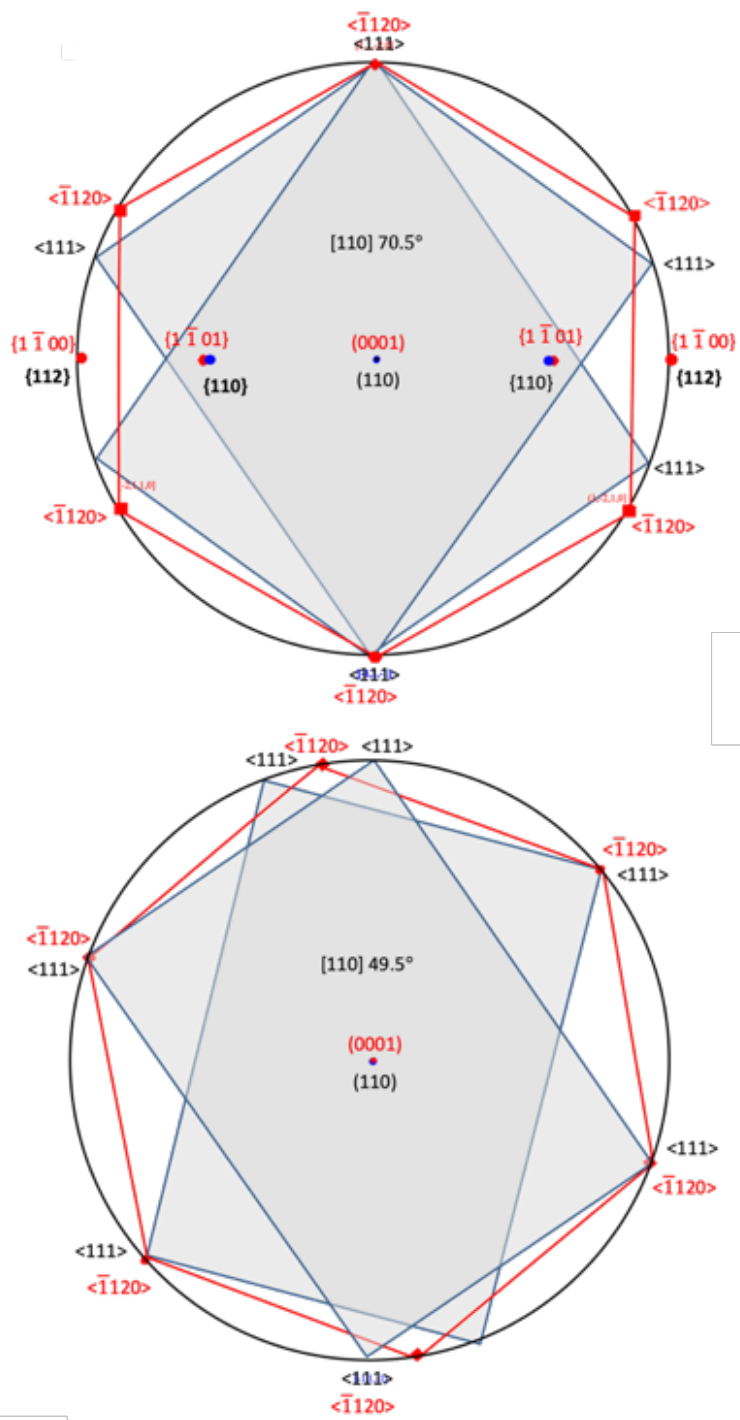

a
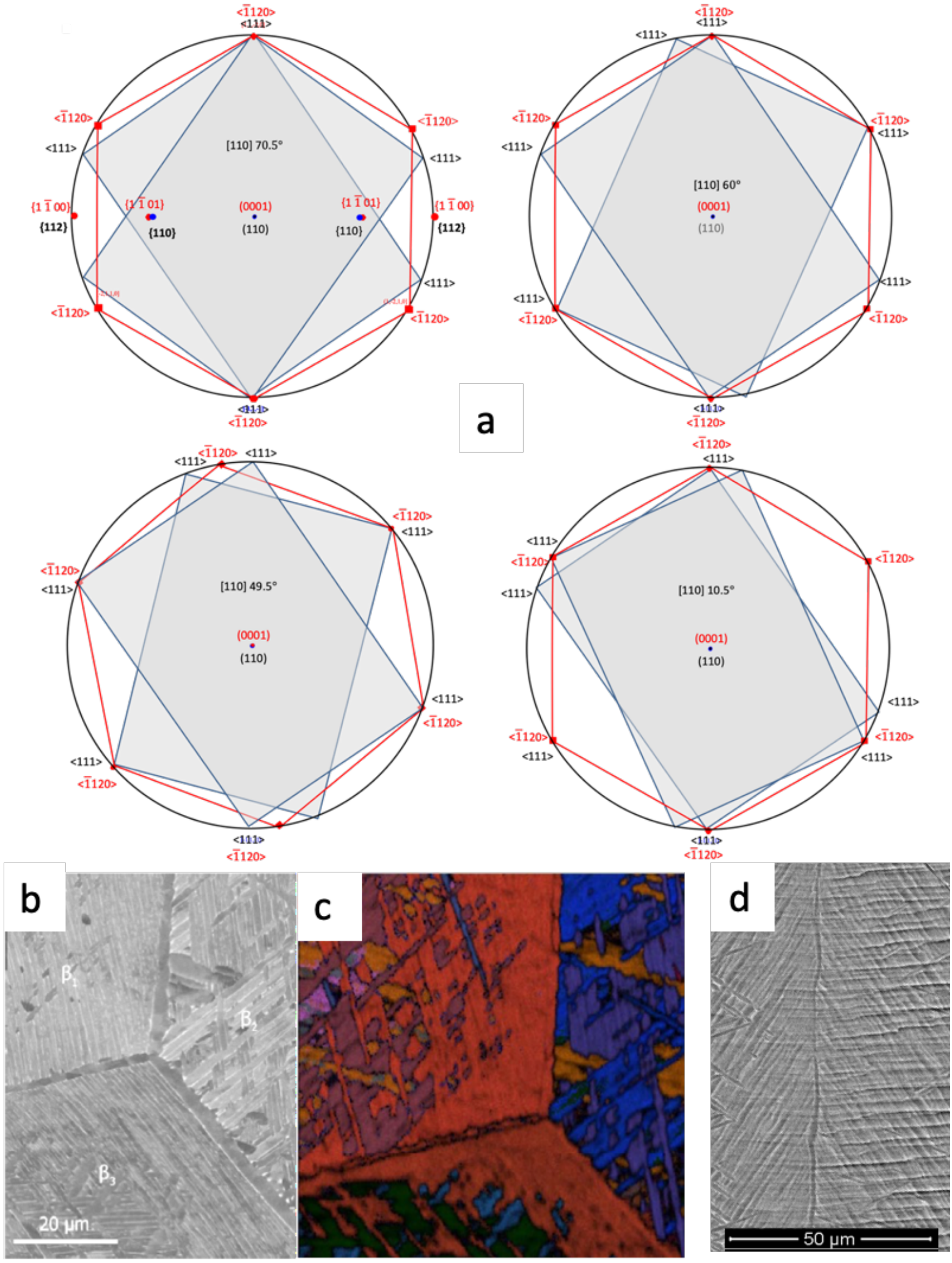

Figure 14: (a) pole figures show the 4 special misorientations between $\beta$ grains that can arise from a $\beta$ to $\alpha$ (as during epitaxial recrystallisation). $\alpha$ phase formed at the boundaries of such grains will be in the Burgers relationship to both grains, as shown by poles in red. In the case of the $[110] 70.5^{\circ} /<111>60^{\circ}$ misorientation both grains share parallel slip planes and a common Burgers vector as marked on the figure (micrograph courtesy Adam Pilchak) (b) and (c) show that grain boundary $\alpha$ and widmanstatten sideplates emanating from grain boundary $\alpha$ into each neighbouring grain have a common orientation for the grains marked $\beta 1$ and $\beta 3$, as expected in the case of a special misorientation between $\beta$ grains (d) slip transfer at $\beta$ grain boundary separating $\beta$ grains with a $[110] 70.5^{\circ}$ misorientation. (b) and (c) are from Pilchak, Banerjee and Williams, unpublished research, 2009, Ohio State University, USA 
sideplates originate from grain boundary $\alpha$ and extend into $\beta$ grains to which it is Burgers related, these sideplates extend into both adjacent grains with these specific misorienations (Figure 14 b,c). A second consequence arises in the unique case where the adjacent $\beta$ grains are related by the special misorientation $<111>$ $60^{\circ}$ (Figure 14a). The rotation axis is a common slip direction in both $\beta$ grains and the close packed direction in $\alpha$, and the symmetry about this rotation axis in relation to the Burgers orientation relationship with $\alpha$ ensures that slip planes in the two adjacent grains and the grain boundary $\alpha$ with this Burgers vector will be parallel or very nearly so. Figure $14 \mathrm{c}$ shows that in this specific case, slip can transfer from one $\beta$ grain to another across the grain boundary $\alpha$ and its sideplates.

\section{Summary}

Many years ago Burgers in a classic contribution demonstrated the relationship between the crystal structures of hcp and bcc phases that is expressed as (110) $\beta / /(0001) \alpha ;(1 \overline{12}) \beta / /(1 \overline{1} 00) \alpha ;[1 \overline{1} 1] \beta / /[11 \overline{2} 0] \alpha$. These planes and directions are also the slip planes and slip vectors in the two phases. Additionally, the orientation relationship places pyramidal slip planes in $\alpha$ closely parallel to $\{110\}$ slip planes in $\beta$ for the specific c/a ratio of titanium. The magnitudes of the slip vectors along the close packed directions of $\alpha$ and $\beta$ are quite similar. $\alpha / \beta$ titanium alloys are constituted by relatively coarse microstructures of these two phases that are not substantively different in their individual yield strengths. Thus slip transfer between the two phases occurs relatively easily, albeit constrained by the inherent crystallographic assymetry of the orientation relationship [8,18]. As shown in this contribution, slip lengths or the mean free path for slip in these two-phase microstructures are then determined by the variety of two-phase distributions whose characteristics are dominated by the energetics associated with the Burgers orientation relationship, whether these transformations occur at grain boundaries, the interior of grains or during thermomechanical processing and recrystallisation. A key issue in crystal plasticity modelling of such complex two-phase distributions is to determine a representative volume element (or a set of RVE) that will be representative of the material as a whole (see [19,20] for example). Definitions of RVE vary. In the context of $\alpha / \beta$ alloys it is possible that microstructural units as defined by the mean free path for slip in the specific two-phase distributions may provide such a representation. It is also possible that such a descriptor will provide additional clarity on the physics underlying advanced neural network models for the mechanical behaviour of titanium alloys [21].

\section{Acknowledgements}

Funding from Boeing Companay with support from KK Sankaran and Jim Cotton, Pratt and Whitney, USA with support from Rajiv Naik, Vasisht Venkatesh and Dave Furrer, and from AOARD/AFOSR, USA with Jaimey Tiley and Jeremy Knopp as contract monitors is gratefully acknowledged. One of the authors DB is also grateful for the Raja Ramanna fellowship of DAE, India and the JC Bose Fellowship of DST, India.

\section{References}

[1] D. Choudhuri, T. Borkar, R. Banerjee, D. Banerjee, Proceedings of the 13th World Conference on Titanium, Ed. V Venkatesh et al, TMS (The Minerals, Metals \& Materials Society), pp 515-519.

[2] S. Balachandran, A. Kashiwar, A. Choudhury, D. Banerjee, R. Shi, Y. Wang, Acta Mater. 106(2016) 374387.

[3] S. Balachandran, S. Kumar, D. Banerjee, Acta Mater. 131(2017) 423-434.

[4] A. Banerjee, S. Balachandran, S. Suwas, D. Banerjee, Proceedings of the 13th World Conference on Titanium, Ed. V Venkatesh et al, TMS (The Minerals, Metals \& Materials Society), pp 1095-1101.

[5] A. Banerjee, Masters Thesis, Indian Institute of Science, Bangalore, India, 2015

[6] M. Chinara, Masters Thesis, Indian Institute of Science, Bangalore, India , 2018

[7] D. Banerjee, J.C. Williams, Defence Science Journal, 36(1986) 191-206.

[8] S. Suri, G.B. Viswanathan, T. Neeraj, D.-H. Hou, M.J. Mills, Acta mater. 47 (1999) 1019-1034.

[9] J. Luster, M. A. Morris, Metall. Mater. Trans. A 26 (1995) 1745-1756. 
[10] H. Margolin, P. Cohen, Titan. 80, Conf. Proc. 4th Int. Conf. Titan., TMS, Warrendale, PA, 1980, H. Kimura, O. Izumi (Eds.), pp. 1555-1561.

[11] I. Weiss, F.H. Froes, D. Eylon, G.E. Welsch, Metall. Trans. A, 17 (1986) 1935-1947.

[12] R.D. Doherty, D.A. Hughes, F.J. Humphreys, J.J. Jonas, D.J. Jensen, M.E. Kassner, W.E. King, T.R. McNelley, H.J. McQueen, A.D. Rollett, Materials Science and Engineering A238 (1997) 219-274.

[13] L. Germain, N. Gey, M. Humbert, P. Bocher, M. Jahazi, Acta Mater. 53 (2005) 3535-3543.

[14] E. Uta, N. Gey, P. Bocher, M. Humbert, J. Gilgert, J Microsc. 233(2009) 451-459.

[15] C. Cayron, Scripta Mater. 59 (2008) 570-573.

[16] T. Liu, L. Germain, J. Teixeira, E. Aeby-Gautier and N. Gey, Acta Mater. 141 (2017) 97-108.

[17] D. Bhattacharyya, G.B. Viswanathan, H.L. Fraser, Acta Mater. 55 (2007), 6765-6778.

[18] D. Banerjee, J.C. Williams, Acta Mater. 61 (2013) 844-879.

[19] S. M. Qidwai, D. M. Turner, S.R. Niezgoda, A. C. Lewis, A.B. Geltmacher, D.J. Rowenhorst, S.R. Kalidindi, Acta Mater. 60 (2012) 5284-5299.

[20] M.P Echlin, W.C. Lenthe, T. M Pollock, Integrating Materials and Manufacturing Innovation 2014, 3-21.

[21] I. Ghamarian, P. Samimi, V. Dixit, P. C. Collins, Metall. Mater. Trans. A 46 (2015) 5021-5037. 